\title{
FIXED POINT THEOREMS IN PRODUCT SPACES
}

\author{
TADEUSZ KUCZUMOW
}

(Communicated by Paul S. Muhly)

\begin{abstract}
If $K_{1}$ and $K_{2}$ are nonempty closed weakly compact subsets of Banach spaces and they have the generic fixed point property for nonexpansive mappings, then in the maximum norm $K_{1} \times K_{2}$ has fixed point property for nonexpansive mappings.
\end{abstract}

In this paper we study fixed points of nonexpansive mappings in product spaces.

Let $\left(K, \zeta_{1}\right)$ and $\left(L, \zeta_{2}\right)$ be metric spaces. A mapping $T: K \rightarrow L$ is nonexpansive if

$$
\zeta_{2}(T x, T y) \leq \zeta_{1}(x, y)
$$

for all $x, y \in K$. Let $\chi$ denote the Hausdorff (or ball) measure of noncompactness in $K$ and $L$. A continuous mapping $T: K \rightarrow L$ is a $k$-set contraction $k \in[0,1))$, if for any $K_{0} \subset K$ we have

$$
\chi\left(T K_{0}\right) \leq k \chi\left(K_{0}\right) .
$$

$\left(K, \zeta_{1}\right)$ has a fixed point property for nonexpansive (continuous) mappings if every nonexpansive (continuous) self-mapping $T: K \rightarrow K$ must have a fixed point.

Let $K$ be a nonempty, convex, weakly compact subset of a Banach space $X . K$ has the generic fixed point property for nonexpansive mappings if for every nonexpansive self-mapping $T: K \rightarrow K$ and every nonempty, convex, closed subset $K_{0} \subset K$ with $T K_{0} \subset K_{0}$, we have $K_{0} \cap \operatorname{Fix}(T) \neq \varnothing$, where $\operatorname{Fix}(T)=\{x \in K: T x=x\}$.

Suppose that $\left(X,\|\|_{1}\right)$ is a Banach space and $\left(K_{2}, \zeta_{2}\right)$ is a metric space. Let $X \times K_{2}$ denote a product space of $X$ and $K_{2}$ with a maximum metric

$$
\zeta_{\infty}((x, u),(y, v))=\max \left(\|x-y\|_{1}, \zeta_{2}(u, v)\right)
$$

for $x, y \in X$ and $u, v \in K_{2}$. It was shown in [7] (see also [2, 5, 6, 8, 9, 10]) that if $X$ has $K K$-norm, $\varnothing \neq K_{1} \subset X$ is weakly compact and convex, and $K_{1}$ and $K_{2}$ have the fixed point property for nonexpansive mappings, then every

Received by the editors Mand 3 , 1984 .

1980 Mathematics Subject Cleceffication (1985 Rexision). Primary 47H09, 47H10.

Key words and phrases. Nopexpansive mappings, nonexpansive retracts, fixed points. 
nonexpansive (with respect to the metric $\zeta_{\infty}$ ) $\quad T: K_{1} \times K_{2} \rightarrow K_{1} \times K_{2}$ has a fixed point. Here we generalize this result. We begin with

Theorem 1. Let $\left(X,\|\|_{1}\right)$ be a Banach space and let $\left(K_{2}, \zeta_{2}\right)$ be a metric space. Suppose that $\varnothing \neq K_{1} \subset X$ is weakly compact, convex and has the generic fixed point property for nonexpansive mappings. If $F: K_{1} \times K_{2} \rightarrow K_{1}$ is a nonexpansive mapping, then there exists a nonexpansive mapping $R: K_{1} \times K_{2} \rightarrow K_{1}$ such that

$$
F(R(x, u), u)=R(x, u)
$$

for $(x, u) \in K_{1} \times K_{2}$ and $R(x, u)=x$ when $F(x, u)=x$.

Proof. We construct a mapping $\tilde{F}: K_{1} \times K_{2} \rightarrow K_{1} \times K_{2}$ in the following way

$$
\tilde{F}(x, u)=(F(x, u), u)=\left(F, P_{2}\right)(x, u)
$$

for $(x, u) \in K_{1} \times K_{2}$, where $P_{2}$ is the second coordinate projection. It is trivial to check that $\tilde{F}$ is nonexpansive and $A=\operatorname{Fix}(\tilde{F}) \neq \varnothing$. Now we define the set $N(A)=\left\{\tilde{G}=\left(G, P_{2}\right) \in K_{1} \times K_{2}^{K_{1} \times K_{2}}: G: K_{1} \times K_{2} \rightarrow K_{1}\right.$ is nonexpansive and $A \subset \operatorname{Fix}(\tilde{G})\}$.

Note that

$$
N(A) \subset \prod_{(x, u) \in K_{1} \times K_{2}} K_{1} \times\{u\} \sim \prod_{(x, u) \in K_{1} \times K_{2}} K_{1} .
$$

In $K_{1}$ we have the weak topology, so by Tychonoff's Theorem $\prod_{(x, u) \in K_{1} \times K_{2}} K_{1}$ is compact in the product topology. This topology can be transposed on the set $\prod_{(x, u) \in K_{1} \times K_{2}} K_{1} \times\{u\}$ and $N(A)$ is closed in this topology. It allows us to apply Bruck's method ([1]) step-by-step to obtain a nonexpansive retraction $r: K_{1} \times K_{2} \rightarrow A$ which additionally belongs to $N(A)$, i.e. $r=\left(R, P_{2}\right) . R$ satisfies the desired conditions.

Let $X, K_{1}, K_{2}$ be as in Theorem 1. Suppose that $T=\left(T_{1}, T_{2}\right): K_{1} \times K_{2} \rightarrow$ $K_{1} \times K_{2}$ is a mapping such that $T_{1}: K_{1} \times K_{2} \rightarrow K_{1}$ is nonexpansive. Fix $x_{0} \in K_{1}$. The mapping $\bar{T}_{2}: K_{2} \rightarrow K_{2}$ is defined by

$$
\bar{T}_{2}(u)=T_{2}\left(R\left(x_{0}, u\right), u\right), \quad u \in K_{2},
$$

where $R$ satisfies Theorem 1 . It is clear that $\bar{T}_{2}(u)=u$ implies that $T\left(R\left(x_{0}, u\right), u\right)=\left(R\left(x_{0}, u\right), u\right)$. Let $K_{2}$ have the fixed point property with respect to a subclass $S$ of $K_{2}^{K_{2}}$. If $\bar{T}_{2} \in S$, then $\operatorname{Fix}(T) \neq \varnothing$.

Theorem 2. Let $X, K_{1}, K_{2}$ be as in Theorem 1 and let $T_{1}: K_{1} \times K_{2} \rightarrow K_{1}$ be nonexpansive.

(i) If $\left(K_{2}, \zeta_{2}\right)$ has a fixed point property for nonexpansive mappings and $T_{2}: K_{1} \times K_{2} \rightarrow K_{2}$ is nonexpansive, then $T=\left(T_{1}, T_{2}\right): K_{1} \times K_{2} \rightarrow K_{1} \times K_{2}$ has a fixed point.

(ii) If $\left(K_{2}, \zeta_{2}\right)$ has a fixed point property for continuous mappings and $T_{2}: K_{1} \times K_{2} \rightarrow K_{2}$ is continuous, then $T=\left(T_{1}, T_{2}\right): K_{1} \times K_{2} \rightarrow K_{1} \times K_{2}$ has a fixed point. 
(iii) If $\varnothing \neq K_{2}$ is a convex, closed subset of a Banach space $X$ and $T_{2}: K_{1} \times K_{2} \rightarrow K_{2}$ is a $k$-set contraction with respect to Hausdorff measure of noncompactness, then $T=\left(T_{1}, T_{2}\right): K_{1} \times K_{2} \rightarrow K_{1} \times K_{2}$ has a fixed point.

Proof. It is sufficient to observe that in the first case $S$ is the set of all nonexpansive self-mappings in $K_{2}$, in the second one $S$ is the set of all continuous self-mappings in $K_{2}$, and in the third one $S$ is the set of all $k$-set contractions in $K_{2}$.

Remark 1. If $K_{1}, \ldots, K_{n}$ are nonempty, convex, weakly compact subsets of Banach spaces and have the generic fixed point property for nonexpansive mappings, then $\prod_{i=1}^{n} K_{i}$ has a fixed point property for nonexpansive mappings (with respect to the maximum norm).

Remark 2. For discussion of spaces which have the fixed point property with respect to nonexpansive mappings, we refer to Kirk and $([3,4])$ and Reich $([11$, 12]).

Remark 3. If $X_{1}$ is the conjugate Banach space, then in the above theorems the weak topology can be replaced by the weak-* topology.

\section{REFERENCES}

1. R. E. Bruck, Properties of fixed point sets of nonexpansive mappings in Banach spaces, Trans. Amer. Math. Soc. 179 (1973), 251-262.

2. A. Fora, A fixed point theorem for product spaces, Pacific J. Math. 99 (1982), 327-335.

3. W. A. Kirk, Fixed point theory for nonexpansive mappings, Fixed Point Theory (E. Fadell and G. Fournier, eds.), Lecture Notes in Math., no. 886, Springer-Verlag, New York, 1981, pp. 484-505.

4. __ Fixed point theory for nonexpansive mappings II, Fixed Points and Nonexpansive Mappings (R. C. Sine, ed.), Contemp. Math. 18 (1983), 121-140.

5. _ Nonexpansive mappings in product spaces, set-valued mappings and $k$-uniform rotundity, Nonlinear Functional Analysis and its Applications (F. E. Browder, ed.), Amer. Math. Soc. Symp. Pure Math., Vol. 45, 1986, pp. 51-64.

6. __ Fixed points theorems in product spaces, Operator Equations and Fixed Points (S. P. Singh, V. M. Seghal, and J. H. W. Burry, eds.), Math. Sci. Res. Inst. Korea 1 (1986), 27-35.

7. __ An iteration process for nonexpansive mappings with applications to fixed point theory in product spaces, Proc. Amer. Math. Soc. (to appear).

8. W. A. Kirk and C. Martinez, Nonexpansive and locally nonexpansive mappings in product spaces, Nonlinear Anal. 12 (1988), 719-725.

9. W. A. Kirk and Y. Sternfeld, The fixed point property for nonexpansive mappings in certain product spaces, Houston J. Math. 10 (1984), 207-214.

10. S. Nadler, Jr., Sequences of contractions and fixed points, Pacific J. Math. 27 (1968), 579-585.

11. S. Reich, The fixed point property for nonexpansive mappings I, Amer. Math. Monthly 83 (1976), 226-228.

12. $\ldots$, The fixed point property for nonexpansive mappings II, Amer. Math. Monthly 87 (1980), 292-294.

Instytut Matematyki UMCS, Plac Maril Curie-Sklodowskiej 1, 20-031 Lublin, Poland 